\title{
Avaliação da qualidade de vida de idosos com e sem correção de valvulopatia cardíaca: relatos de casos
}

\author{
valvulopatas elderly valve heart life \\ quality
}

FisiSenectus . Unochapecó Ano 2, n. 1 - Jan/Jun. 2014 p. $43-50$

Lilian Marin . fisiolili@unochapeco.edu.br

Mestre em Envelhecimento Humano, Docente do Curso de Fisioterapia da Universidade Comunitária da Região de Chapecó (Unochapecó).

Sabrina de Lima .sabrinalima@unochapeco.edu.br Estudante do Curso de Graduação em Fisioterapia, Universidade Comunitária da Região de Chapecó (Unochapecó).

Leticia Giacomin . lety_giacomin@unochapeco.edu.br

Estudante do Curso de Graduação em Fisioterapia, Universidade Comunitária da Região de Chapecó (Unochapecó).

\section{Resumo}

Objetivo: Avaliar a qualidade de vida de idosos com e sem correção de valvulopatia cardíaca. Método: Trata-se de múltiplos estudos de caso em que foram utilizados os seguintes instrumentos para avaliar a qualidade de vida: MacNew QLMI (composto por 27 questões divididas entre domínios físico, social e emocional); WHOQOL-bref (composto 26 questões divididas em domínios físico, psicológico, social e ambiental); e uma ficha sociodemográfica contendo: dados pessoais, diagnóstico clínico, história familiar, hábitos de vida e doenças crônicas associadas. Foram avaliados quatro idosos, três homens (que realizam fisioterapia regularmente) e uma mulher (não realiza fisioterapia), com idade entre 64 e 75 anos, dos quais somente dois realizaram correção cirúrgica de valvulopatia aórtica, um homem e uma mulher. Resultados: Após a análise dos dados verificou-se que, a doença crônica mais presente foi a Hipertensão Arterial Sistêmica observada em todos os idosos avaliados, seguido pelo IAM em três idosos. Quanto aos hábitos de vida, três dos pacientes foram tabagistas. Quanto à análise do MacNew QLMI e WHOQOL-bref para ambos os instrumentos observou-se que o domínio físico prevaleceu melhor para três idosos, sendo que um passou por correção cirúrgica. Para um idoso prevaleceu o domínio psicológico, este também passou por correção cirúrgica. Conclusão: Observa-se, nesses casos, que a correção cirúrgica influenciou diretamente na percepção de qualidade de vida no domínio físico do instrumento WHOQOL-bref, já que estes apresentaram escores menores nesse domínio. 0 grau de acometimento clínico não influenciou na qualidade de vida dos indivíduos. Seria interessante uma amostra maior para que as conclusões fossem mais precisas.

\section{Palavras-chave}

Geriatria, Cardiopatia, Indicadores de Qualidade de vida.

\section{Fisiß̌enectus}




\begin{abstract}
Goal: To evaluate the elder quality of life with and with our correction of Cardiac valvulopathy. Method: This is the case of multiple studies that the instruments used to evaluate the quality of life: MacNew QLMI (Consists of 27 questions divided bet we en are as physical, social and emotional); WHOQOL-bref (Comprising 26 questions divided into are as physical, psychological, social and environmental); and a sociodemographic connect or containing: personal data Clinical diagnosis, family history, life style and associated chronic diseases. Wee valuated four elderly, three men (that perform physiotherapy regularly) and a woman (not performs physical therapy), with age between 64 and 75 years, where only two underwent surgical correction of aortic valvular disease a man and a woman. Results: After analysis off the it was found that chronic disease more prevalent was the Hypertension Systemic Arterial observed in all elderly patients evaluated; followed by AMI in three elderly, dyslipidemia in two and in the habits of life, two of them were smokers. Regarding the analysis of the Mac New QLMI and WHOQOL-bref for both instruments it was observed that the physical domain prevailed better for three elderly, being that one of these has gone through surgical correction and for an elderly person who has prevailed the psychological domain, knowing that this also started by surgical correction. Conclusion: in these cases, that the surgical correction had direct influence on the perception of quality of life in the physical domain of the instrument WHOQOL-bref, since these had lower scores in this area. The degree of clinical impairment does not influence the quality of life of individuals. It would be interesting to perform a larger ample for conclusions were more accurate.
\end{abstract}

\title{
Keywords
}

Geriatrics, Heart disease, Indicators of Quality of life.

\section{Introdução}

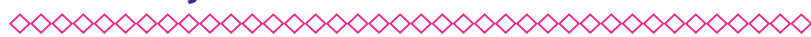

O envelhecimento populacional é um fenômeno mundial. No Brasil, segundo dados do Instituto Brasileiro de Geografia e Estatística (IBGE), amparado pela maior expectativa de vida, o número de brasileiros acima de 65 anos deve praticamente quadruplicar até 2060, confirmando a tendência de envelhecimento acelerado da população. As doenças ligadas ao processo do envelhecimento levam ao dramático aumento dos custos assistenciais de saúde ${ }^{1}$.

Estudos com base populacional têm demonstrado que, em nosso país, a maioria dos idosos são portadores de pelo menos uma enfermidade crônica². No Brasil, o número de idosos com idade superior a 60 anos passou de sete milhões em 1975 para quatorze milhões em 2002 e vinte milhões em 2010 e estima-se que em 2020 esse número será de trinta e dois milhões ${ }^{3}$.

0 avançar da idade, por si só, aumenta o risco de doenças crônicas, com destaque para as cardiovasculares. Apesar de aumentar com a idade, grande parte dessas doenças poderia ser evitada, assim como as doenças crônicas mais frequentes (cardiovasculares, câncer e diabetes) que com- partilham vários fatores de risco. A Organização Mundial de Saúde (OMS) propõe uma abordagem de prevenção e controle integrado, em todas as idades, baseada na redução dos seguintes fatores: Hipertensão Arterial Sistêmica (HAS), fumo, álcool, inatividade física, dieta inadequada, obesidade e hipercolesterolemia².

As valvulopatias cardíacas são a terceira causa mais frequente de insuficiência cardíaca congestiva no idoso, subsequente à isquemia miocárdica e à hipertensão arterial sistêmica ${ }^{4}$. Reconhecer a doença valvar no idoso é difícil, devido à complexidade do quadro, caracterizado por manifestações atípicas e pela presença de doenças associadas. A história clínica nem sempre apresenta os sintomas com precisão cronológica devido às múltiplas doenças que compreendem a dificuldade do idoso em expressar seus sintomas. Com o curso da doença, os sintomas podem se intensificar, com a possibilidade do surgimento de dispneia paroxística noturna, edema agudo de pulmão e hemoptise. Outras vezes a doença permanece silenciosa, sendo reconhecida clinicamente por início súbito, devido ao quadro de insuficiência cardíaca ${ }^{5}$.

As formas de tratamento para as valvulopatias cardíacas são: o tratamento conservador que envolve tanto a parte de controle dos sinto- 
mas, administração de medicamentos, fisioterapia e o tratamento cirúrgico, dependendo do grau de acometimento $^{6-8}$. Sabe-se que esses pacientes acabam tendo interferência na sua qualidade de vida. As doenças cardiovasculares podem diminuir a qualidade de vida dos indivíduos portadores de valvulopatia, pois podem causar um comprometimento importante tanto na saúde física quanto na mental. Isso porque a doença valvar pode gerar alterações na capacidade física, mental e perceptiva dos indivíduos, reduzindo, assim, a qualidade de vida.

No que se refere ao conceito qualidade de vida, na literatura, podemos encontrar várias formas de abordagem, portanto não há um consenso estabelecido. 0 conceito de qualidade de vida está relacionado à autoestima e ao bem-estar pessoal e abrange uma ampla variedade de aspectos, tais como: capacidade funcional, estado emocional, nível socioeconômico, interação social, estado de saúde, valores culturais, religiosidade, estilo de vida, entre outros. Sendo assim, o conceito qualidade de vida é subjetivo e dependente do nível sociocultural da idade e das ecessidades pessoais de cada indivíduo 9 .

É importante ressaltar que para observar a qualidade de vida existem diversos instrumentos de avaliação na forma de questionários e entrevistas, os quais abordam as condições de vida e saúde de uma forma ampla. Dentre estes podemos citar: SF36, WHOQOL, MINESSOTA, MacNew QLMI, entre outros.

Com base nesse contexto, a avaliação da qualidade de vida em saúde em idosos com valvulopatias cardíacas é de suma importância, visto que possibilita identificar elementos que podem ser modificados e que interferem na percepção individual da qualidade de vida. Diante disso, o objetivo deste estudo foi: avaliar como está a qualidade de vida de pacientes idosos com e sem correção cirúrgica de valvulopatias.

\section{Método}

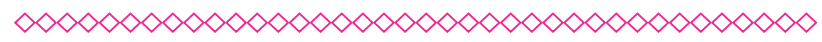

A pesquisa foi composta por estudos de caso múltiplos, realizados em âmbito ambulatorial nos municípios de Xanxerê-SC e Chapecó-SC. A amos- tra foi composta por idosos valvulopatas submetidos ou não a cirurgia valvar, de ambos os gêneros e com idade acima de 60 anos. Quanto aos critérios de inclusão temos: idosos que aceitassem participar do estudo, assinando o termo de consentimento livre e esclarecido, e que apresentassem condições físicas e psíquicas para responder a entrevista. Já para os critérios de exclusão: não estarem em condição clínica que impossibilite responder a entrevista.

Após a aprovação do Comitê de Ética e Pesquisa da Universidade Comunitária de Chapecó-SC, parecer $n^{0}$ 1.128.807, contatou-se idosos submetidos ou não à correção de valvulopatia cardíaca explicando sobre o objetivo e o procedimento de coleta de dados deste estudo. Em sequência agendou-se as entrevistas, sendo apenas uma com cada participante. Os participantes que aceitaram e assinaram o termo de consentimento livre esclarecido, tiveram suas entrevistas agendadas em local de sua preferência, onde foram submetidos a uma avaliação. Para coleta de dados utilizou-se uma ficha sociodemográfica contendo: dados pessoais, diagnóstico clínico, história familiar, hábitos de vida, doenças crônicas associadas, pressão arterial de repouso (PA), frequência cardíaca ( $F C)$, saturação de oxigênio (SatO2), peso, estatura e índice de massa corporal (IMC). Na sequência, os participantes responderam a uma entrevista referente a dois instrumentos de avaliação de qualidade de vida Mac New QLMI e WHOQOL-bref com duração média de 20 minutos. A coleta foi realizada no mês de junho de 2015.

0 instrumento Mac New QLMI é constituído por 27 questões com escore mínimo de 1 e máximo de 7. Sendo assim, o escore total máximo corresponde a 189 e o mínimo a 27.0 instrumento é dividido em três domínios, o escore físico é calculado pela soma de 14 itens, o escore emocional pela soma de 14 itens e o escore social pela soma de 13 itens. Esse questionário é aplicado para pacientes pós-infarto, porém, no presente estudo, foi adaptado para pacientes pré e pós correção de valvulopatia ${ }^{10-12}$. Já o WHOQOL-bref que é um instrumento genérico composto por 26 questões, sendo duas gerais e as demais representando cada uma das 24 facetas que formam o instrumento original. 0 questionário é dividido em quatro domínios: físico, psicológico, relações sociais e meio ambiente, as 
respostas seguem uma escala de Likert (de 1 a 5 , quanto mais alto o escore, melhor a qualidade de vida), porém não há ponto de corte para sua classificação ${ }^{13}$

Após o término da pesquisa, analisou-se de forma descritiva cada caso, utilizando os instrumentos em cada domínio. O WHOQOL-bref foi utilizado para o domínio físico, em que foi realizado o somatório das questões e a soma dividida por sete. Já para o domínio psicológico foi realizado o somatório das questões e a soma dividida por seis. Para o domínio relações sociais foi realizado o somatório e este foi dividido por três. Por fim, para o domínio ambiental, foi realizado o somatório e este dividido por oito. Para o Mac New QLMI foi realizado somente a soma de cada domínio físico, emocional e social, considerando que quanto maior a pontuação, melhor a percepção do indivíduo quanto à sua qualidade de vida.

\section{Resultados}

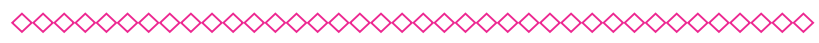

A amostra foi composta por quatro indivíduos. No início da pesquisa haviam cinco indivíduos envolvidos, contudo, um foi excluído devido não ter o seu diagnóstico clínico preciso de valvulopatia cardíaca.

Para organização dos resultados os indivíduos foram organizados em casos, divididos quanto ao seu perfil (Tabela 1) e quanto à análise da qualidade de vida (Tabela 2 e 3).

Estudo de caso 1: Indivíduo de sexo masculino, 75 anos, com diagnóstico de insuficiência aórtica moderada. Doenças crônicas associadas: HAS, IAM e bronquite asmática. Não é tabagista. Não possui história familiar para doenças cardíacas. Realiza fisioterapia (reabilitação cardiopulmonar) duas vezes por semana com duração de 50 minutos cada sessão.

Estudo de caso 2: Indivíduo de sexo masculino, 73 anos, tem com diagnóstico de estenose e insuficiência mitral leve, insuficiência aórtica leve, insuficiência tricúspide mínima. Não realizou correção de válvula aórtica. Possui doenças crônicas associadas: Diabetes, HAS. Relata que foi tabagista, mas cessou o vício há aproximadamente 40 anos. Não possui história familiar. Realiza fisioterapia duas vezes por semana durante 50 minutos cada sessão.

Estudo de caso 3: Indivíduo de sexo masculino, 75 anos, realizou correção de válvula aórtica (há 20 anos). Doenças crônicas associadas: HAS, IAM, dislipidemia, distúrbio vascular periférico e obesidade. Cessou o tabagismo há trinta anos, porém não soube informar por quanto tempo perdurou o vício. Possui história familiar de mãe e irmão cardiopatas com IAM. Realiza fisioterapia duas vezes por semana durante 50 minutos cada sessão.

Estudo de caso 4: Indivíduo de sexo feminino, 64 anos, realizou correção de válvula aórtica (há dois anos). Possui doenças crônicas associadas: HAS, IAM, dislipidemia, distúrbio vascular periférico e obesidade. Cessou o tabagismo há três anos, porém não soube informar a quanto tempo foi tabagista. Possui história familiar de mãe e irmão cardiopatas, mas também não soube informar precisamente qual a cardiopatia. Não realiza fisioterapia e nenhum outro tipo de exercício físico.

Quanto à análise de qualidade de vida verificada pelo instrumento Mac New QLMI, percebeu-se que o domínio físico, emocional e social dos indivíduos 2 e 3 apresentaram escores com melhor qualidade de vida (Tabela 2).

Já no Whoqol bref verificou-se que o domínio físico dos indivíduos 3 e 4 que realizaram correção de valvulopatia cardíaca apresentaram menor escore. Quanto aos domínios psicológico, ambiental e social, os indivíduos 2 e 3 apresentam maior escore, comparado com os demais, sendo que estes são os que possuem a idade mais elevada (Tabela 3).

A partir da análise dos dados pôde-se observar que três pacientes eram do sexo masculino e um do sexo feminino. Todos os pacientes possuem HAS e três deles apresentam também diagnóstico de IAM. Ainda é possivel observar que 0 paciente 3 e o paciente 4 possuem dislipidemia, distúrbio vascular periférico, obesidade e história familiar de cardiopatas, sendo que esses dois pacientes foram submetidos ao procedimento de correção de válvula aórtica. Três dos entrevistados foram tabagistas, porém cessaram o vício, e três dos quatro pacientes realizam fisioterapia regularmente. 
No instrumento Mac New QLMI foi possível observar que três dos quatro indivíduos apresentaram melhores escores no domínio físico do que nos domínios emocional e social. No questionário WHOQOL-bref o domínio social apresentou maior qualidade de vida em todos os indivíduos, já o domínio físico apresentou escores mais baixos nos indivíduos 3 e 4, ambos que já realizaram procedimento cirúrgico.

Deve ser ressaltado que a percepção de qualidade de vida tanto no domínio psicológico e social do questionário WHOQOL-bref, quanto nos domínios emocional e social no questionário Mac New QLMI, podem variar muito dependendo do momento em que os questionários são aplicados, pois fatores como dor, uma noite mal-dormida ou problemas pessoais estão diretamente ligados a esses domínios, o que pode acabar interferindo na escolha das respostas.

\section{Discussão}

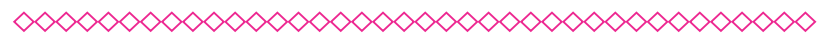

O termo qualidade de vida sempre esteve ligado a melhorias no padrão de vida, associado a bens materiais adquiridos. A qualidade de vida incorpora-se na sensação de bem-estar, realização pessoal, qualidade dos relacionamentos, estilo de vida, educação, saúde, enfim, aspectos físicos, psicológicos e sociais, além dos econômicos. Sendo assim, o conceito qualidade de vida é muito amplo e inclui aspectos objetivos e subjetivos ${ }^{14}$.

Na área da saúde, especificamente na cardiologia, o conceito qualidade de vida pode variar de acordo com o tipo de procedimento realizado pelo paciente, bem como com o momento em que a pergunta foi realizada. Segundo Grinberg, ${ }_{1}^{15} \mathrm{em}$ clínica de valvopatia, cabe bem a associação da classe funcional de I a IV com a avaliação objetiva nomeada de A a D. Um portador de insuficiência aórtica grave, por exemplo, sem dispneia, cansaço, palpitação ou angina no peito durante as atividades diárias é considerado com capacidade funcional de classe I e avaliação objetiva $D$; e, sem nenhuma alteração de variáveis como ausculta do sopro e bulhas, diâmetros e função ventricular esquerda entre o dav before e dav after, a conduta, em algum momento, precisa ser mudada. 0 mais significativo, nesse caso, é o sintoma, a queixa referida do portador de valvopatia. Esse é o ponto-chave que o paciente nos disponibiliza e, após a nossa interpretação, resulta ao encaixe correto à classe funcional, sendo, então, um receptor de conduta terapêutica ${ }^{16,17}$.

0 estudo, avaliado de acordo com métodos previamente estabelecidos, e as entrevistas, realizadas com pacientes que foram ou não submetidos à correção valvar, apontou elevadas semeIhanças entre os itens dos questionários Mac New QLIM e WHOQOL-bref. Enquanto o Mac New QLMI tem enfoque na percepção do paciente, o WHOQOL-bref foi elaborado para avaliar a percepção do paciente de uma forma global, com relação a diferentes aspectos de sua vida. Em todos os entrevistados, os questionários aplicados apresentaram maior qualidade de vida no domínio social. 0 domínio físico apresentou escores mais baixos nos indivíduos que já realizaram correção cirúrgica de valvulopatia cardíaca. Observou-se também que a perspectiva de qualidade de vida mais alta encontra-se nos pacientes que apresentam idade mais elevada.

Em estudo realizado por Gonçalves et al. ${ }^{17}$ sobre a qualidade de vida na reabilitação cardíaca, observou que na primeira avaliação pós-operatória os pacientes apresentaram declínio nos domínios do SF-36, porém, quando foi realizada a segunda avaliação, entre dois e seis meses após o procedimento cirúrgico, a pontuação em todos os domínios havia sido normalizada.

Segundo Barros et al., ${ }^{18}$ analisando os maiores e menores escores dos questionários entre os domínios avaliados pelo SF-36, é possível encontrar o pior resultado em aspectos físicos, seguido pelos aspectos emocionais, e o melhor em aspectos sociais. Assim, os escores mais baixos demonstraram uma qualidade de vida prejudicada em percepção da saúde.

Estudos realizados por Ferreira et al. ${ }^{19}$ sobre a qualidade de vida em pacientes submetidos à cirurgia valvular cardíaca apontam que a qualidade de vida dos doentes depois de serem submetidos à cirurgia valvular é melhor do que a qualidade de vida dos doentes antes de realizarem o procedimento. É possível observar essa melhoria em todas as dimensões, exceto nas dimensões função social 
e desempenho físico. A idade não teve influência na percepção da qualidade de vida para os dois grupos (pré e pós-operatório). Os doentes, antes de serem operados, apresentam um conjunto de limitações físicas decorrentes da doença cardíaca superior aos doentes já submetidos à cirurgia valvular. Observou-se que no grupo pré-operatório os doentes que se apresentavam em uma classe funcional I e II da NYHA, manifestavam melhores escores nas dimensões função física, saúde geral e saúde mental do que os indivíduos que se encontravam em uma classe funcional III e IV.

Custódio e Gasparino20 realizaram estudos com 38 indivíduos que estavam em pós-operatório de cirurgia cardíaca, foram aplicados o WHOQOL-Bref e o questionário sociodemográfico. Os resultados obtidos foram de que os sujeitos, em sua maioria, eram do sexo masculino, casados e que não completaram o ensino fundamental. Os escores do WHOQOL-Bref foram: domínio físico $(62,9)$, psicológico $(76,1)$, social $(74,3)$, ambiental $(69,2)$, avaliação geral da qualidade de vida $(75,0)$ e satisfação com a saúde $(73,7)$, concluindo, assim, que os participantes apresentaram elevada percepção da qualidade de vida, comparados a outros estudos. A aplicação de questionários referentes à avaliação da QV em pacientes portadores de doenças cardíacas tem se mostrado de grande utilidade na avaliação dos resultados alcançados com esse tipo de tratamento ${ }^{19}$.

Os indivíduos incluídos no estudo não foram homogêneos quanto ao tempo, gravidade da doença e realização ou não de reabilitação cardiovascular. As variações nas medidas dos escores podem estar relacionadas às atividades da vida diária que esses pacientes realizavam, que não foram avaliadas nesta pesquisa, além de fatores socioeconômicos que podem influenciar quanto à percepção de qualidade de vida de cada indivíduo envolvido no estudo. Cabe ressaltar aqui que há escassez de estudos sobre qualidade de vida em indivíduos com valvulopatias cardíacas.

\section{Conclusão}

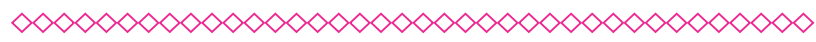

Com os dados analisados pôde-se concluir que os indivíduos avaliados apresentaram HAS como principal fator de risco cardiovascular, seguido pelo IAM, porém, fatores como obesidade, distúrbio vascular periférico e dislipidemia foram encontrados apenas nos pacientes que foram submetidos à correção de valvulopatia aórtica. 0 número restrito dos avaliados demonstrou parâmetros pequenos na amostra, o que impede uma interpretação mais detalhada do comportamento da qualidade de vida nesses indivíduos. Observa-se, nesses casos, que a correção cirúrgica teve influência direta na percepção de qualidade de vida no domínio físico do instrumento WHOQOL-bref, já que apresentaram escores menores nesse domínio. 0 grau de acometimento clínico não influenciou na qualidade de vida dos indivíduos. Seria interessante a análise de amostras maiores para obtenção de conclusões mais precisas. Porém as informações adquiridas podem contribuir na elaboração de estudos futuros com diferentes grupos envolvidos e uma amostra maior de avaliados.

\section{Agradecimentos}

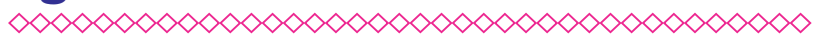

Aos indivíduos, por aceitarem participar da pesquisa e nos permitir realizar este estudo, colaborando no fornecimento dos dados necessários.

\section{Referências}

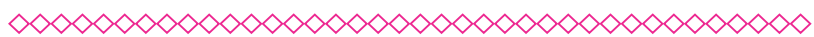

\section{Ponciano MJ. Aspectos divergentes nos} discursos gerontológicos. Caderno geração, ano 5 , $n^{0} 6$, abril 2015.

2. Pereira JC, Barreto SM, Passos VMA.O perfil de saúde cardiovascular dos idosos brasileiros precisa melhorar: estudo de base populacional. Arq. Bras. Cardiol. 2008 Jul 91; (1): 1-10.

3. Bezerra FC, Almeira MI, Nobrega SM. Estudos sobre envelhecimento no Brasil: revisão bibliográfica. Rev. bras. geriatr. gerontol. 2012; 15( 1 ): 155-167.

4. Archer SL, Chesler E. Diagnosis of Valve Disease in the Elderly. In: Chesler E. Clinical Cardiology in the Elderly. 2nd Edition: Futura Publishing Company, 1999. 
5. Hara JH. Valvular Heart Disease. Primary Care, 2000.

6. Regena MM. Fisioterapia em cardiologia: da UTi á reabilitação. São Paulo: Roca, 2000.

\section{Zocrato LBR, Machado MGR. Fisioterapia} Respiratória no Pré e Pós-Operatório de Cirurgia Cardíaca. In: Machado MGR. Bases da Fisioterapia Respiratória: Terapia Intensiva e Reabilitação. Rio de Janeiro: Guanabara Koogan; 2008.

8. Ridley SC. Cirurgia em Adultos. In: Pryor JA, Webber BA. Fisioterapia para Problemas Respiratórios e Cardíacos. $2^{\mathrm{a}}$ ed. Rio de Janeiro: Guanabara Koogan; 2010.

9. Dawalibi NW, et al. Envelhecimento e qualidade de vida: análise da produção científica da SciELO. Estudos de Psicologia (Campinas). 2013; 30(3), 393-403.

10. Lim Lly, et al. A self-administered quality of life questionnaire after acute myocardial infarction. J Clin Epidemiol. 1993 Nov;46(11):1249-56.

11. Oldridge N, Guyatt G, Crowe J, Feeny D, Jones $\mathrm{N}$. Goal attainment in a randomized controlled trial of rehabilitation after myocardial infarction. J Cardiopulm Rehabil. 1999 Jan-Feb;19(1):29-34.

12. Benetti M, Nahas MV, Barros MVG. Reproducibility and validity of a brazilian version of the Mac New quality of life after myocardial infarction (Mac New QLMI) questionnaire. Medicine \& Science in Sports \& Exercise, 2001.

13. Fleck MPA, Chachamovich, Trentini, CM. WHOQOL-OLD Project: method and focus group results in Brazil. Rev. Saúde Pública. 2003 37( 6 ): 793-799.

14. Seidl EMF, Zannon CMLC. Qualidade de vida e saúde: aspectos conceituais e metodológicos. Cad. Saúde Pública. 2004 20( 2 ): 580-588.

15. Grinberg M. Prescindindo da história natural, decidindo-se pela história pós-operatória na estenose valvar aórtica: uma releitura bioética. Rev. Soc. Cardiol. 2003 13(3):378-388.
16. Tarasoutchi F, et al. Ten-year clinical laboratory follow-up after application of a symptom-based therapeutic strategy to patients with severe chronic aortic regurgitation of predominant rheumatic etiology. J Am Coll Cardiol. 2003 Apr 16;41(8):1316-24.

17. Gonçalves FDP, et al. Avaliação da qualidade de vida pós-cirurgia cardíaca na fase I da reabilitação através do questionário MOS SF-36. Rev. bras. fisioter. 200610 ( 1 ): 121-126.

18. Barros RT, et al. Avaliação de aspectos da qualidade de vida em pacientes pós-implante de marca-passo cardíaco. Rev Bras Cir Cardiovasc. 2014 29( 1 ): 37-44.

19. Ferreira AJS, et al. Qualidade de vida em doentes submetidos a cirurgia valvular cardíaca. Psic., Saúde \& Doenças. 2008 9( 1 ): 155-164.

20. Custódio FM, Gasparino, RC. Qualidade de vida de pacientes no pós-operatório de cirurgia cardíaca. Rev. Min. Enferm. 2013 17(1):126-135. 


\section{Anexos}

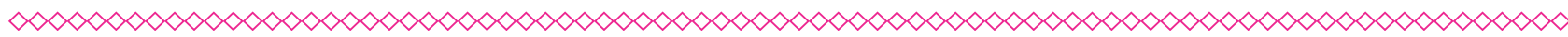

Tabela 1 - Perfil da amostra

\begin{tabular}{|c|c|c|c|c|}
\hline Variáveis & Individuo 1 & Indivíduo 2 & Indivíduo 3 & Indivíduo 4 \\
\hline Idade (anos) & 75 & 73 & 75 & 64 \\
\hline Gênero (M/F) & $M$ & $M$ & $M$ & $\mathrm{~F}$ \\
\hline Peso (Kg) & 67,40 & 64 & 87,50 & 80 \\
\hline Estatura (m) & 1,80 & 1,60 & 1,75 & 1,62 \\
\hline IMC $\left(\mathrm{Kg} / \mathrm{m}^{2}\right.$ & 24,17 & 25 & 28,57 & 30,40 \\
\hline $\mathrm{PA}(\mathrm{mmHg})$ & $140 \backslash 90$ & $100 \backslash 60$ & $120 \backslash 80$ & $130 \backslash 90$ \\
\hline $\mathrm{FC}(\mathrm{bpm})$ & 76 & 96 & 92 & 85 \\
\hline Sat02 (\%) & 98 & 95 & 96 & 96 \\
\hline $\begin{array}{l}\text { Correção Cirúrgica (sim/ } \\
\text { não) }\end{array}$ & não & não & $\operatorname{sim}$ & $\operatorname{Sim}$ \\
\hline
\end{tabular}

Fonte: Dados da pesquisa, 2015.

(clique para voltar ao texto)

Tabela 2 - Análise Mac New QLMI

\begin{tabular}{|c|c|c|c|c|}
\hline Domínios & Indivíduo 1 & Indivíduo 2 & Indivíduo 3 & Indivíduo 4 \\
\hline Físico & 71 & 85 & 89 & 84 \\
\hline Emocional e Social & 58 & 89 & 80 & 71 \\
\hline
\end{tabular}

Fonte: Dados da pesquisa, 2015.

(clique para voltar ao texto)

Tabela 3 - Análise Whoqol-Bref

\begin{tabular}{|c|c|c|c|c|}
\hline Domínios & Indivíduo 1 & Indivíduo 2 & Indivíduo 3 & Indivíduo 4 \\
\hline Físico & 3,42 & 4,14 & 3,28 & 3,14 \\
\hline Psicológico & 2,83 & 4,16 & 5,00 & 3,33 \\
\hline Ambiental & 3,25 & 4,62 & 5,00 & 4,00 \\
\hline Social & 3,60 & 5,00 & 4,33 & 4,00 \\
\hline
\end{tabular}

Fonte: Dados da pesquisa, 2015.

(clique para voltar ao texto) 